\title{
Design and CFD Analysis of Micro-Fin Heat Sink
}

\author{
${ }^{1}$ Mahendra M \\ Department of Mechanical \\ Engineering \\ Islamiah Institute of Technology \\ Bangalore-560076, India
}

\author{
${ }^{2}$ Salma Fathima \\ Department of Mechanical \\ Engineering \\ Islamiah Institute of Technology \\ Bangalore-560076, India
}

\author{
${ }^{3}$ Mohammed Mathenulla Shariff \\ Associate Professor \\ Department of Mechanical \\ Engineering \\ Islamiah Institute of Technology \\ Bangalore-560076, India
}

\author{
${ }^{4}$ Altafahmed M K \\ Assistant Professor \\ Department of Mechanical Engineering \\ Islamiah Institute of Technology \\ Bangalore-560076, India
}

\author{
${ }^{5}$ Nisar Pasha \\ Assistant Professor \\ Department of Mechanical Engineering \\ Islamiah Institute of Technology \\ Bangalore-560076, India
}

\begin{abstract}
A numerical study has been carried out to investigate the heat transfer enhancement and fluid flow characteristics for various materials of rectangular micro channel heat sinks (MCHS). The working fluid considered for the analysis is Air. The fluid flow and heat transfer characteristics of a three-dimensional MCHS are obtained numerically by solving the appropriate governing equations. Analysis is developed for a conjugated heat transfer model for the study flow and heat transfer in Micro-Fin Heat Sink Array of 10 and 15 number of fins made up of 3 different materials viz Alluminium, Copper and Steel. For further discretization of flow domain, ANSYS Fluent 14.5 CFD code is used in the form of finite volume elements. Further study is carried to simulate the results for different types of fin Numbers with different material configurations, to predict heat transfers prior to effect of heat sink geometry, effect of heat flux and fluid and effect of fin materials.
\end{abstract}

Keywords - Micro - Fin, Heat Sink, Heat Transfer, CFD, ANSYS FLUENT.

\section{INTRODUCTION}

In the present era, the trend to design electronic products becomes thinner, lighter, shorter and smaller. Due to the actuality that shrinking in the dimension of these electronic components will consequence in a drastic increase in the heat generation rate when evaluating with previous products. Technology involving high speeds of data processing in microprocessors of fast computers has led to reduction in sizes of integrated chips. The need to remove large amount of heat generated from such devices for effective functional characteristics. For this reason, an efficient cooling system to remove the high heat generation and consequently maintains the reliability and stability of the products, have gained much attention. Now a day, electronic devices shows the tendency for smaller, faster and denser chips with higher heat transfer rate. As the chip becomes more powerful and smaller, thermal management of the chips becomes more complicated. As the density of the transistor increases the heat flux from the system increases. Electronic devices require more effective thermal enhancement system to maintain its operating temperatures at a satisfactory level due to the increase of power density.
A heat sink is a passive heat exchanger that transfers the heat generated by an electronic or a mechanical device to a fluid medium, often air or a liquid coolant, where it is dissipated away from the device, thereby allowing regulation of the device's temperature at optimal levels. In computers, heat sinks are used to cool central processing units or graphics processors. Heat sinks are used with highpower semiconductor devices such as power transistors and optoelectronics such as lasers and light emitting diodes (LEDs), where the heat dissipation ability of the component itself is insufficient to moderate its temperature.

A heat sink is designed to maximize its surface area in contact with the cooling medium surrounding it, such as the air. Air velocity, choice of material, protrusion design and surface treatment are factors that affect the performance of a heat sink. Heat sink attachment methods and thermal interface materials also affect the die temperature of the integrated circuit. Thermal adhesive or thermal grease improve the heat sink's performance by filling air gaps between the heat sink and the heat spreader on the device. A heat sink is usually made out of copper or aluminium. Copper is used because it has many desirable properties for thermally efficient and durable heat exchangers. First and foremost, copper is an excellent conductor of heat. This means that copper's high thermal conductivity allows heat to pass through it quickly. Aluminium heat sinks are used as a low-cost, lightweight alternative to copper heat sinks, and have a lower thermal conductivity than copper.

Extended surface or fins are commonly used to enhancement of heat transfer from a system to surrounding any fluid. The fins are improving the heat transfer from system to surrounding fluid. The application of fins has been investigated for different purpose such as electronics cooling, industrial process and energy generation process. In a convective heat transfer, there are two ways such as increasing area or increase the heat transfer coefficient. For increase heat transfer coefficient there is need of fan or blower. It is more cost than natural convection. Natural convection is done by using the increase the surface area and also the there is no space for the fan or blower. The micro fins are having with micro scale with very small dimension. Also, the fin having various types like straight fin of uniform 
cross section, straight fin of non-uniform cross section, annular fin and pin fin. There are used by depending on application or special purpose.

The material selection for fin is very important depending on the application and also depends on material properties. Fin material having with the high thermal conductivity and also other thermal properties. According to high thermal conductivity Diamond having very high thermal conductivity due to lattice vibration but it is lot expensive than the other material. Also, the copper having with high thermal conductivity $(420 \mathrm{~W} / \mathrm{mK})$ is best but copper is expensive. It is used for special purpose application. The aluminum and its alloy are the best material for the fin with having high thermal conductivity (219 $\mathrm{W} / \mathrm{mK})$.

\section{WORKING PRINCIPLE}

A heat sink transfers thermal energy from a higher temperature device to a lower temperature fluid medium. The fluid medium is frequently air, but can also be water, refrigerants or oil. If the fluid medium is water, the heat sink is frequently called a cold plate. In thermodynamics a heat sink is a heat reservoir that can absorb an arbitrary amount of heat without significantly changing temperature. Practical heat sinks for electronic devices must have a temperature higher than the surroundings to transfer heat by convection, radiation, and conduction. The power supplies of electronics are not $100 \%$ efficient, so extra heat is produced that may be detrimental to the function of the device.

As such, a heat sink is included in the design to disperse heat.

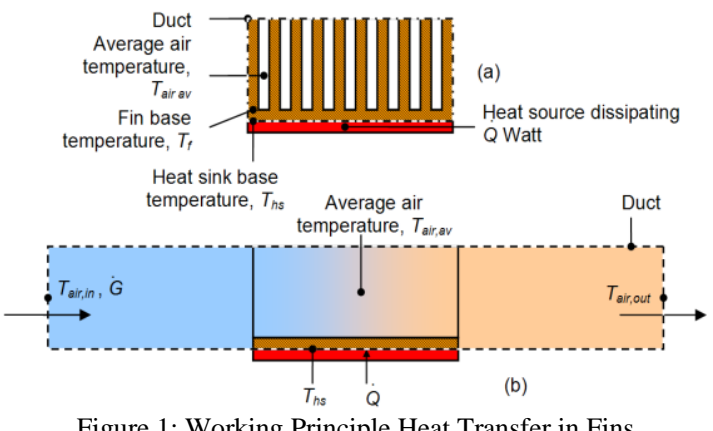

\section{LITERATURE REVIEW}

C.J. Kobus, T. Oshio 2005 [1] investigate the effect of thermal radiation on the thermal performance of heat sink having pin fin array by theoretical and experimental approach. In order to investigate the ability of influence of thermal radiation on the thermal performance a new coefficient effective radiation heat transfer is collaborated. For validation of theoretical model it is matched with experimental data.

Hung-Yi Li et al. 2007 [2] done their investigation on platefin heat sinks numerically and experimentally. Impingement cooling is used by amending, the Reynolds number (Re), the impingement distance (Y/D), and the fin dimensions. The results shows that heat transfer is enhance by the heat sink with increasing the impinging Reynolds number.
G. Hetsroni et al. 2008, [12] Natural convection heat transfer in metal foam strips with two porosities examined experimentally. Image processing of the thermal maps were used for evaluation of nonequilibrium temperature distribution for surface along with inner area of the metal foam. Augmentation in heat transfer at natural convection was found 18-20 times with respect to the flat plate.

Goshayeshi and Ampofo 2009 [3] conduct numerical studies on vertical fins, attached with the surface. Natural convective heat transfer find out from heated plane, which is kept into air with horizontal and vertical surface. Results show that vertical plate with dimensionless form delivers best performance for the natural cooling.

Dong-Kwon Kimet al. (2009) [4] compared the thermal performances of two types of heat sinks i.e.: plate-fin and the second is pin-fin. By their investigation results propose, a volume averaging approach-based model for envisaging the pressure drop and the thermal resistance. Burak and Hafit 2009 [5] developed expression for prediction of the optimal fin spacing for vertical rectangular fins with rectangular base. The correlation for predicted on the basis of experimental data. $\mathrm{Li}$ and Chao 2009 investigated the performance of platefin heat sinks with cross flow. The effect of different parameters like the fin width, fin height, Re. number of cooling air on the thermal resistance and the pressure drop of heat sinks were studied. Naidu et al.2010 investigates by both experimentally and theoretically to find the outcome of inclination of the base of the fin array on heat transfer rate.

Sable et al. 2010 [6] investigated the natural convection of a vertical heated plate with a multiple $v$ - type fins having ambient air surrounding. The mica gladded Nichrome element is inserted between two base plates. Mahmoud et al. 2011 conducted an experiment to investigate the effects of micro fin height and spacing on heat transfer coefficient for a horizontally mounted heat sink under steady state natural convection conditions, fin height ranging from $0.25-1.0 \mathrm{~mm}$ and fin spacing from 0.5 to $1.0 \mathrm{~mm}$ was taken. Cheng-Hung al. 2011 developed a three-dimensional heat sink design to estimate the optimum design variables. LevenbergMarquardt Method (LMM) was used and commercial code CFD-ACE+ was developed. Temperature distributions are dignified by using thermal camera for the optimal heat sink modules and results are compared with the numerical solutions to validate the design.

Fahiminia et al. 2011 [7] investigated the laminar natural convection on vertical surfaces computationally. The CFD simulations are carried out using fluent software. Governing equations are solved using a finite volume approach. Relation between the velocity and pressure is made with SIMPLE algorithm Ayla dogan et al. 2012 performed numerical investigation to find out the natural convection heat transfer from an annular fin on a horizontal cylinder and present correlation for the optimum fin spacing depending on Rayleigh number and fin diameter. Mateusz al.2013 used water and copper oxide nano fluids for cooling heat sink of PC Processor. 
The commercial package ANSYS Fluent 13 was employed to generate a CFD heat transfer simulation. The experimental results were used to validate the numerical model of the analyzed system.

Farhad et al.2013 [8] solved .Navier-Stokes equations and RNG based k- turbulent model for array of solid and perforated fins mounted in vertical flat plates used to predict turbulent flow parameters. Flow and heat transfer features are presented for Re. no. from $2 \times 104-3.9 \times$ 104.Prandtl numbers was taken as 0.71. Numerical simulation is validated by compare with experimental results. Qarnia and Lakhal 2013 using numercail approach investigate the heat transfer by natural convection during the melting of a phase change material. A mathematical model was developed to investigate the thermal performance of PCM based-heat sink.

Mehran al. 2014 [9] examined numerically and experimentally, Steady-state external natural convection heat transfer from vertically-mounted rectangular interrupted fins. FLUENT software is employed to develop a 2-D numerical model of fin interruption effects. An experimental numerical parametric study was performed to investigate the effects of fin spacing, and fin disruption. A new compact correlation is proposed for calculating the optimum interruption length. Emrana and Islama 2014 performed a three-dimensional numerical simulation is in order to investigate the flow dynamics and heat transfer characteristics in a microchannel heat sink.

Chen et al. (2005) [10] presented a method for thermal optimization of a pin fin heat sink under multiple constraints like the available pressure drop, the space limitations and the mass restrictions. In the proposed approach: (a) statistical methods were applied for the sensitivity analysis of the design factors, (b) the design of experiments (DOE) and the response surface methodology (RSM) were used for the development of regression models of the thermal resistance and pressure drop in terms of the design factors and (c) the gradientbased numerical optimization technique was employed in the search for optimal design parameters.

Hung et al. [11] had investigated the effect of different configurations of porous micro channel heat sinks on their thermal performance. They had analyzed six various configurations of porous heat sinks and ended up with sandwich configuration which yielded better thermal performance. They mainly concluded that the porous micro channel heat sinks need not be efficient over the non-porous micro channel heat sinks at low pumping power.

Lin et al. [12] had studied the effect of double layer micro channel heat sink and their thermal performance numerically for a three-dimensional geometry. The working fluid was water and they had examined six different types of double layer heat sinks.

They had optimized the dimension of the double layer heat sink for a particular volumetric flow rate and pumping power and they had reported that the double layer heat sink should be examined for every particular application as it varies for different working conditions and could not be generalized.

Xie et al. [13] had studied recently about the obstruction induced in the path of the micro channel heat sink. They reported that the boundary layers get destroyed and new boundary layers have been built which aid in the thermal performance of the heat sinks. They evaluated that obstruction length plays a dominant role in thermal performance of the MCHS.

Latest researches are going on, in investigating the effect of magnetic influence, porous medium and nanoparticles in enhancing the heat transfer characteristics of liquid coolants used for various applications including MCHS and have been briefly discussed as follows.

Sheikholeslami et al. [14] had carried out an intensive research on the influence of magnetic field on the copper oxide water nano fluid flow and heat transfer characteristics using Lattice Boltzmann method. KooKlein streuer-Li correlation was employed to calculate the thermal conductivity and viscosity of the working fluid.

They concluded that the heat transfer characteristics increases with increase in Hartmann number and heat source length and decreases with Rayleigh number.

Zeeshan et al. [15] had worked on water and ethylene glycol based nanofluid heat transfer characteristics with an inverted cone, by varying the wall temperature and predicted the natural convective boundary layer flow and magnetic effect using nonlinear ordinary differential equation employing Boussinesq approximation. They derived a correlation for skin friction and heat transfer rate for the above model which incorporates porous medium also.

Zeeshan and Majeed [16] has critically examined the Non-Darcy boundary layer flow of non-conducting real fluid. The analysis was carried with magnetic ferro particles over a permeable linearly stretching surface taking into account the Ohmic dissipation along with mixed convective heat transfer.

They converted the existing governing partial differential equations into nonlinear ordinary differential equations by similarity transformation. They concluded that the skin friction coefficient increases and Nusselt number decreases with increase in ferro magnetic interaction parameter.

Ellahi et al. [18] had recently analyzed the impact of shapes of nano-fluid on entropy generation by developing a mathematical model analytically using Boussinesq approximation. They investigated the natural convection boundary layer flow over an inverted cone by considering simultaneously the power law effects, porous medium, magnetohydrodynamics and radiation effects in their study.

\section{PROBLEM DEFINITION}

Due to rapid evolution in a wide range of technologies in twentieth century, heat dissipation requirement has increased very rapidly especially from compact systems. There is an urgent need for high-performance heat sinks to ensure the integrity and long life of these petite 
systems. Use of forced convection cooling has been limited by the requirement of the excessively high flow velocity and associated noise and vibration problems. Along with the rapid growth in electronic industry, applications of miniaturisation are immense in past decades. The miniaturization, however, inevitably generates excessive amount of heat on the highly limited surface area. It is indeed crucial to develop an efficient cooling method, particularly when the conventional heat sink is inadequate to meet the high heat flux requirements.

Liquid-cooled microchannel heat sinks and coolers have been shown to be a very effective way to remove high heat load. A large heat transfer coefficient can be achieved by reducing the channel hydraulic diameter. In a confined geometry the small flow rate within microchannels produces laminar (smooth) flow, which results in a heat transfer coefficient inversely proportional to the hydraulic diameter. In other words, the narrower the channels in the heat sink, the higher the heat transfer coefficient. Heat dissipation is an unavoidable by-product of electronic devices and circuits.

In general, the temperature of the device or component will depend on the thermal resistance from the component to the environment, and the heat dissipated by the component. To ensure that the component does not overheat, a thermal engineer seeks to find an efficient heat transfer path from the device to the environment.

\section{Objectives}

\section{OBJECTIVES AND METHODOLOGY}

$>$ To carry out numerical study to investigate the heat transfer enhancement and fluid flow characteristics for various aspect ratios of rectangular micro channel heat sinks.

$>$ To develop a conjugated heat transfer model for the study flow and heat transfer in Micro-Fin Heat Sink Array of 10 and 15 number of fins made up of 3 different materials viz Alluminium, Copper and Steel.

$>$ To discretize flow domain as finite volume elements and solve using ANSYS Fluent 14.5. Hence further on to extend the CFD code to study the influence of geometrical parameters.

$>$ To simulate the results for different types of fin Numbers with different material configurations, to predict heat transfers prior to effect of heat sink geometry, effect of heat flux and fluid and effect of fin materials.

\section{Methodology}

$>$ Micro-Fins Heat Sinks are designed separately as per the required dimensions using SolidWorks design tool.

$>$ The iges file format of the Heat Sinks file is later imported to ICEM CFD 14.5 tool to carry out Hexa meshing by capturing the $y+$ value effectively.
$>$ Once the meshing is done, later it is converted to unconstructed mesh file format and is imported to ANSYS Fluent 14.5 Solver.

$>$ In Fluent, all the necessary solid and fluid flow regimes, boundary conditions and converging residuals limits of mass, momentum and energy has been applied satisfactorily.

$>$ Once solution is converged, later it is imported into CFD Post to carry out the Post Processing Activity.

\section{DESIGN CONSIDERATIONS}

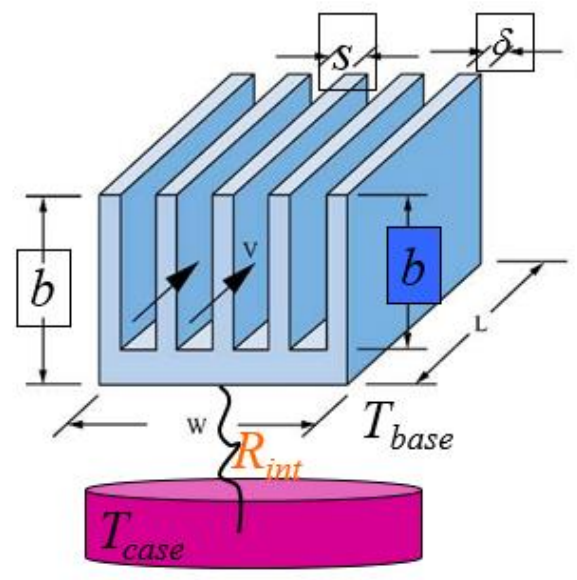

Figure 2: Design Considerations in Fins

In order to select the appropriate heat sink, the thermal designer must first determine the maximum allowable heat sink thermal resistance. To do this it is necessary to know the maximum allowable module case temperature, $\mathrm{T}_{\text {case, }}$ the module power dissipation, $\mathrm{q}_{\text {mod }}$, and the thermal resistance at the module-to-heat sink interface, $R_{\text {int }}$. The maximum allowable temperature at the heat sink attachment surface, $T_{\text {base }}$, is given by

$$
T_{\text {base }}=T_{\text {case }}-q_{\text {mod }} \times R_{\text {int }}
$$

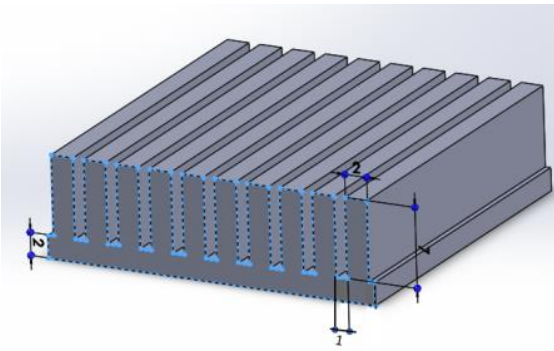

Figure 3: Geometry of 10 No. of Fins Heat Sink

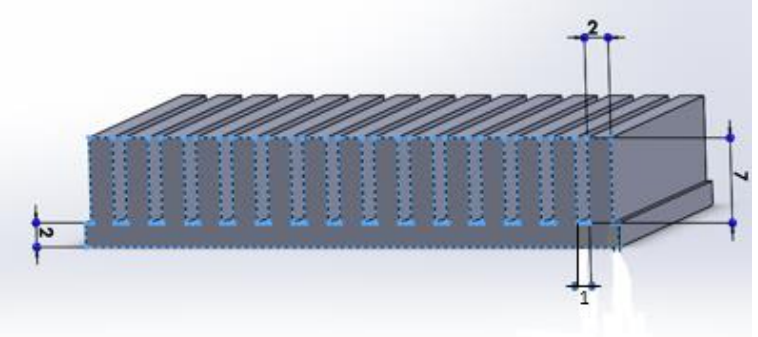

Figure 3: Geometry of 15 No. of Fins Heat Sink 
SOLIDWORKS is used for creation of CAD Data as shown in the figures above. The three-dimensional discretized model is generated in the meshing tool ICEMCFD in the pre-processor phase as shown in Fig. 4. The entire domain is discretized into finite volume hexahedral elements to capture the thermal and hydraulic boundary layers as shown in Fig. 5 by very fine grids near the walls. A y+ value of less than 1 is maintained near the fluid solid interface to capture this phenomenon. The minimum angle and determinant of the hexahedral elements are maintained at $90^{\circ}$ and 1 respectively, which ensures very good quality of the finite volume discretization.

The micro channel has discrete zones as solid and fluid domains. The inlet boundary is velocity inlet and pressure boundary of zero-gauge pressure is applied at the fluid outlet for the exit boundary condition. The interfaces defined enable fluid flow visualization across the MCHS. Since heat transfer through conduction takes place along the copper solid wall, convection between the heated bottom wall surface and the liquid, the meshing is made very fine in order to simulate conjugate heat transfer which is a combination of conductive and convective heat transfers. The heat transfer by radiation is minimal and negligible and hence in this numerical study radiation effects are not considered.

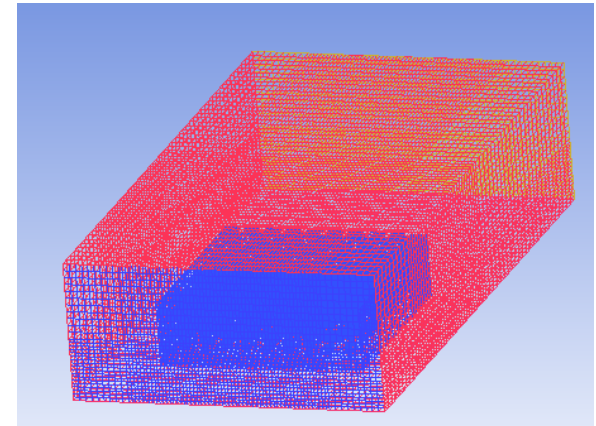

Figure 4: Hexa Mesh of 10 Fin-Fluid Domain in ICEM CFD

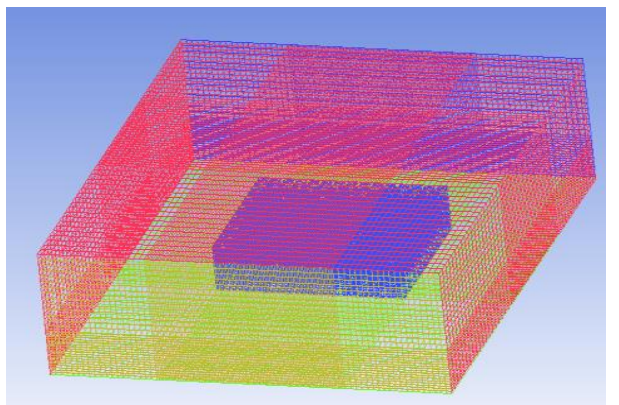

Figure 5: Hexa Mesh of 10 Fin-Fluid Domain in ICEM CFD

The governing equations are the standard continuity equation for conservation of mass, Navier - Stokes equation for conservation of momentum and energy equations for predicting the conjugate heat transfer. The following conditions are assumed in this numerical analysis
$>$ The mass of a fluid is conserved.

$>$ The rate of change of momentum equals the sum of the forces on a fluid particle (Newton's second law).

$>$ The rate of change of energy is equal to the sum of the rate of heat addition to and the rate of work done on fluid particle (first law of thermodynamics).

$>$ Flow is assumed to be laminar and Incompressible.

\section{Boundary Conditions}

The boundary conditions defined in the ANSYSFLUENT processor serve as inputs for the model. The flow considered is laminar. The fluid used in this numerical analysis found to be Air. A constant heat flux of $150 \mathrm{~W} / \mathrm{m}^{2}$ and Temperature of $373 \mathrm{~K}$ are assigned to the bottom wall surfaces. The inlet is velocity $2 \mathrm{~m} / \mathrm{s}$ and is assumed uniform No slip boundary condition is assigned to all the surface. The side and top walls of the MCHS are made insulated. Zero-gauge pressure is assigned at the outlet of the MCHS.

In ANSYS FLUENT solver, the conservation equations are solved at discretized pseudo time steps and the solutions are obtained based on the convergence of mass, momentum and energy equations. An iterative process is carried out by repeated solving of the governing equations; thereby, the errors converge to a bare minimum value up to five decimal places for mass and momentum and up to 7 decimal places for energy which means theoretically almost zero. Thus, the solutions obtained are more reliable with higher values of convergence. The number of elements used for discretizing the domain and the equations selected for fluid flow and conjugate heat transfer greatly influence the quality of the results achieved.

\section{Grid Independency}

The model is tested for grid-independence to give proper resolution to the region where large gradients of fluid flow and heat transfer characteristic is predicted. The fine grid mesh for the $\mathrm{x}$ and $\mathrm{y}$-directions is adopted to properly resolve the velocity and viscous shear layers and to more accurately define the conjugate heat transfer at the surface of the channel, thereby improving the temperature resolution. The meshing along the axial direction away from the entrance region a relatively coarse grid system has taken.

The reasons for the comparative coarse discretization for the z-direction are, with the exception of the inlet region, the temperature gradients are small compared to the gradients occurring in other directions and the CPU time as well as the memory storage required increases dramatically as the number of grid nodes is increased. Furthermore, comparison with standard numerical results, indicates that the finer the mesh size, the higher the numerical accuracy. 


\section{RESULTS AND DISCUSSION}

Conjugate heat transfer simulation work consists of analysis of both convection heat transfer and conduction heat transfer processes. The air (fluid) flows over the fins at the interface regions of both solid and fluid. The main equations are solved for fluid flows are the momentum equations and turbulence-modeling equations. Solution of these NavierStokes equations gives the velocity vectors and Temperature in the fluid flow region.

The energy equation is solved for both fluid (convection heat transfer) and solid (conduction heat transfer) regions to obtain temperature distribution along the length of the fin by applying the interface boundary conditions at the coupled region. The overview of the results of present study involves CFD validation and parametric study conducted for different configuration of fins at a constant velocity $2 \mathrm{~m} / \mathrm{s}$ in a duct. And made comparison with Alluminium, Copper and Steel with 10 and 15 number of fin configuration models.
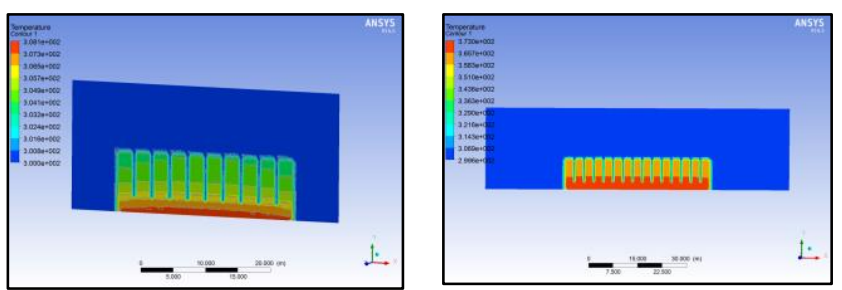

Figure 6: Temperature Counters for Al Fins.
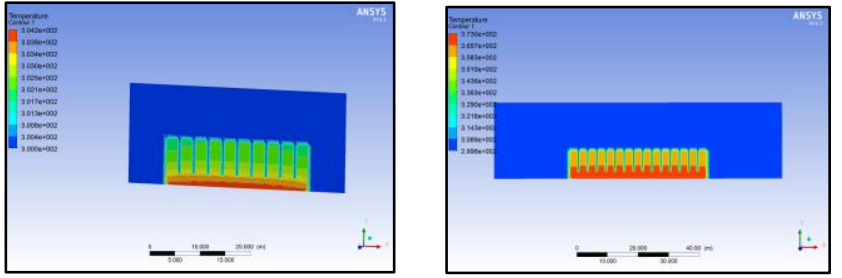

Figure 7: Temperature Counters for $\mathrm{Cu}$ Fins
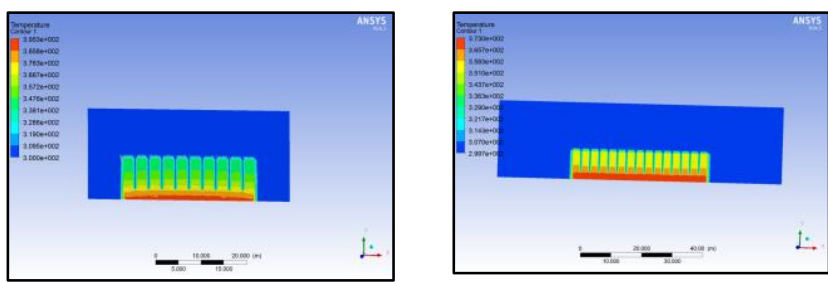

Figure 8: Temperature Counters for Steel Fins.

\section{Effects of heat flux and fluid}

Maximum thermal resistance as a function of heat flux is also investigated here considering water as cooling fluid and assuming the velocity $0.75 \mathrm{~m} / \mathrm{s}$. The heat transfer capability is dependent on the properties of working fluid, the most frequently used coolant in the micro channel heat sink is Air. Better results may be possible with other fluids. So, one of the methods for changing heat transfer is to use the different working fluids.

\section{Effects of heat sink geometry}

It is investigated that the maximum fin temperature is decreased with the increase of the number of fin array. But after a certain increase of array the temperature of the assembly again increases. It is because the coolant does not get enough space to take the heat from the array. The model was also investigated for different fin height and channel width.

\section{Effects of fin material}

The model is also investigated for various types of fin material- Copper, Aluminum and Steel. A MWNT thermal conductivity of $400 \mathrm{~W} / \mathrm{m}-\mathrm{K}$ is used in all simulations. From the simulation it is clearly realized that carbon Copper shows better thermal performance among these materials.

\section{CONCLUSION}

In this work, numerical analysis of different fin geometries of heat sinks for forced convection heat transfer and their comparison carried out by using CFD technique. Different fin geometries were created and effect of heat load on thermal performances are studied and presented through graphs and contours. For all fin geometries and Materials compared for the maximum temperature attained on the basis of result governed by the CFD and conjugative heat transfer analysis.

To find out best heat sink designs, the fin profiles were investigated for enhancing the heat dissipation rate and some thermal improvements as well as space reduction and material savings were attained. Improvements on heat sink designs are possible by the use of CFD. Eventually it is possible to finish up with a new heat sink design which has better thermal performance and uses less material.

$>$ When base surface of fin increases, air present near the surface of fin moves vertically upwards with high velocity.

$>$ In case of high thermal conductivity materials like copper, the thermal boundary layer found to be wider and resulting in higher heat transfer rate.

$>$ In case of Alluminium and Steel, the nonuniformity in temperature and heat flux distribution results in reduction in thermal boundary layer.

$>$ Based on Nusselts Number, with increase in fin spacing, the heat transfer rate also increases.

$>$ Copper often used in high thermal performances in heat transfer applications, alluminium is found to be more appropriate material where mass of the micro-fin is significant.

\section{REFERENCES}

[1] C.J. Kobus , T. Oshio,'Predicting the thermal performance characteristics of staggered vertical pin fin array heat sinks under combined mode radiation and mixed convection with impinging flow" International Journal of Heat and Mass Transfer 48 (2005) 2684-2696

[2] Hung-Yi Li , Kuan-Ying Chen, "Thermal performance of platefin heat sinks under confined impinging jet conditions" International Journal of Heat and Mass Transfer 50 (2007) 1963- 1970

[3] G. Hetsroni, M. Gurevich, R. Rozenblit, "Natural convection in metal foam strips with internal heat generation" Experimental Thermal and Fluid Science 32 (2008) 1740-1747

[4] H. R Goshayeshi, F. Ampofo, "Heat Transfer by Natura Convection from a Vertical and Horizontal Surfaces Using Vertical Fins"Energy and Power Engineering, 2009, 85-89 
[5] Burak YAZICIOĞLU and Hafit YÜNCÜ,"A CORRELATION FOR OPTIMUM FIN SPACING OF VERTICALLY-BASED RECTANGULAR FIN ARRAYS SUBJECTED TO NATURAL CONVECTION HEAT TRANSFER"IS1 BilimiveTekniği Dergisi, 29, 1, 99-105, 2009 J. of Thermal Science and Technology

[6] Hung-Yi Li *, Shung-Ming Chao, "Measurement of performance of plate-fin heat sinks with cross flow cooling" International Journal of Heat and Mass Transfer 52 (2009) 2949-2955

[7] Dong-Kwon Kim, Sung Jin Kim , Jin-Kwon Bae, "Comparison of thermal performances of plate-fin and pin-fin heat sinks subject to an impinging flow", International Journal of Heat and Mass Transfer 52 (2009) 3510-3517

[8] S. V. Naidu, V. Dharma Rao, B. GovindaRao, A. Sombabu and B. Sreenivasulu, "Natural Convection heat transfer from fin arrays-experimental and theoretical study on effect of inclination of base on heat transfer"ARPN Journal of Engineering and Applied Sciences VOL. 5, NO. 9, Sepetember 2010

[9] J. Sable, S.J. Jagtap , P.S. Patil , P.R. Baviskar \& S.B. Barve ,"Enhancement Of Natural Convection Heat Transfer On Vertical Heated Plate By Multiple V-FIN Array"IJRRAS 5 (2) November 2010

[10] Mahdi Fahiminia, Mohammad Mahdi Naserian, Hamid Reza Goshayeshil, Davood Majidian, "Investigation of Natural Convection Heat Transfer Coefficient on Extended Vertical Base Plates"Energy and Power Engineering, 2011, 3, 174-180
[11] Cheng-Hung Huang, Jon-Jer Lu, Herchang Ay , "A three dimensional heat sink module design problem with experimental verification" International Journal of Heat and Mass Transfer 54 (2011) 1482-1492

[12] S. Mahmoud , R. Al-Dadah , D.K. Aspinwall , S.L. Soo , H. Hemida ,"Effect of micro fin geometry on natural convection heat transfer of horizontal microstructures" Applied Thermal Engineering 31 (2011) 627-633

[13] H. El Qarnia , A. Draoui , E.K. Lakhal, "Computation of melting with natural convection inside a rectangular enclosure heated by discrete protruding heat sources" Applied Mathematical Modelling 37 (2013) 3968-3981

[14] Mateusz Korpyś, Mohsen Al-Rashed, Grzegorz Dzido, aJanuszWójcika , "CPU Heat Sink Cooled by Nanofluids and Water Experimental and Numerical Study" Proceedings of the 23rd European Symposium on Computer Aided Process Engineering - ESCAPE 23, June 9-12, 2013, Lappeenranta, Finland

[15] Mehran Ahmadi, Golnoosh Mostafavi, Majid Bahrami, "Natural convection from rectangular interrupted fins"International Journal of Thermal Sciences 82 (2014) 62-71

[16] Md. Emrana, Mohammad Ariful Islama, "Numerical investigation of flow dynamics and heat transfer characteristics in a microchannel heat sink" Procedia Engineering 90 ( 2014 ) $563-568$

[17] Younghwan Joo a, Sung Jin Kim, "Comparison of thermal performance between plate-fin and pin-fin heat sinks in natural convection" International Journal of Heat and Mass Transfer 83 (2015) 345-356. 